UR-1637

UB-HET-01-03

June 2001

\title{
Direct Measurement of the Top Quark Charge at Hadron
}

\section{Colliders}

\author{
U. Baur* \\ Department of Physics, State University of New York, Buffalo, NY 14260, USA \\ M. Buice $^{\dagger}$ \\ Department of Physics, University of Chicago, 5640 S. Ellis Ave., Chicago, IL 60637, USA \\ Lynne H. Orr ${ }^{\ddagger}$ \\ Department of Physics and Astronomy, University of Rochester, Rochester, NY 14627, USA
}

\begin{abstract}
We consider photon radiation in $\bar{t} t$ events at the upgraded Fermilab Tevatron and the CERN Large Hadron Collider (LHC) as a tool to measure the electric charge of the top quark. We analyze the contributions of $\bar{t} t \gamma$ production and radiative top quark decays to $p \stackrel{(-)}{p} \rightarrow \gamma \ell^{ \pm} \nu \bar{b} b j j$, assuming that both $b$-quarks are tagged. With $20 \mathrm{fb}^{-1}$ at the Tevatron, the possibility that the "top quark" discovered in Run I is actually an exotic charge $-4 / 3$ quark can be ruled out at the $\approx 95 \%$ confidence level. At the LHC, it will be possible to determine the charge of the top quark with an accuracy of about $10 \%$.
\end{abstract}

\footnotetext{
*e-mail: baur@ubhex.physics.buffalo.edu

†e-mail: mabuice@uchicago.edu

‡e-mail: orr@pas.rochester.edu
} 


\section{INTRODUCTION}

It is widely believed that the new particle discovered at Fermilab in 1995 [1,2] is the long sought top quark. Its behavior is certainly consistent with Standard Model (SM) expectations for top, but many of its properties are still only poorly known. In particular, the top quark's electric charge - one of the most fundamental quantities characterizing a particle - has not been measured yet. It still remains not only to confirm that the discovered quark has charge 2/3 (and hence the expected SM quantum numbers), but also to measure the strength of its electromagnetic (EM) coupling to rule out anomalous contributions to its EM interactions.

Indeed, one alternative interpretation has not yet been ruled out: that the new particle is a charge $-4 / 3$ quark. In the published top quark analyses of the CDF and D $\varnothing$ Collaborations (see Ref. [3] for a review), the correlations of the $b$-quarks and the $W$ bosons in $\bar{p} p \rightarrow \bar{t} t \rightarrow$ $W^{+} W^{-} \bar{b} b$ are not determined. As a result, there is a twofold ambiguity in the pairing of $W$ bosons and $b$-quarks, and, consequently, in the electric charge assignment of the "top quark" . Besides the Standard Model (SM) assignment, $t \rightarrow W^{+} b,{ }^{\prime \prime} t^{\prime \prime} \rightarrow W^{-} b$ is also conceivable, in which case the "top quark" would actually be an exotic quark with charge $q=-4 / 3$.

Interpreting the particle found at Fermilab as a charge $-4 / 3$ quark is consistent with current precision electroweak data. Current $Z \rightarrow \ell^{+} \ell^{-}$and $Z \rightarrow \bar{b} b$ data can be fit with a top quark of mass $m_{t} \approx 270 \mathrm{GeV}$, provided that the right-handed $b$-quark mixes with the isospin $+1 / 2$ component of an exotic doublet of charge $-1 / 3$ and $-4 / 3$ quarks, $\left(Q_{1}, Q_{4}\right)_{R}$ [ 4 ]. If the top quark would have a mass of $m_{t} \approx 270 \mathrm{GeV}$, it would have escaped detection in Run I of the Tevatron. In this scenario, the particle discovered in Run I is the $Q_{4}$. A direct measurement of the top quark charge would provide a definitive test of this interpretation.

There are several techniques to determine the electric charge of the top quark, $q_{t o p}$, in future collider experiments. At a linear $e^{+} e^{-}$collider, the full set of form factors describing the most general $\gamma \bar{t} t$ vertex function compatible with Lorentz invariance can be probed in $e^{+} e^{-} \rightarrow \bar{t} t$ [5] (similarly for a muon collider). A photon-photon collider would provide even more precision via the two electromagnetic vertices in $\gamma \gamma \rightarrow \bar{t} t$. The status of future lepton and photon colliders is unfortunately still somewhat uncertain, but in the meantime top quarks will be produced copiously at the Fermilab Tevatron and the CERN LHC. At these hadron colliders, $\bar{t} t$ production is so dominated by the QCD processes $\bar{q} q \rightarrow g^{*} \rightarrow \bar{t} t$ and $g g \rightarrow \bar{t} t$ that a measurement of the $\gamma \bar{t} t$ form factors via $q \bar{q} \rightarrow \gamma^{*} \rightarrow \bar{t} t$ is hopeless. Instead, one can measure the top charge by measuring the charges of its decay products, the final state $b$-jets and $W$ bosons. One can further attempt to determine not only the top quark's charge but also the EM coupling strength by directly studying top's electromagnetic interactions through photon radiation in $\bar{t} t$ events.

The first method - measuring the top charge by reconstructing the charges of its decay products - is difficult to realize for $\bar{t} \rightarrow$ all jet decays; however for di-lepton decays, $\bar{t} t \rightarrow \ell^{+} \nu \ell^{-} \bar{\nu} \bar{b} b$, and for semileptonic decays, $\bar{t} t \rightarrow \ell^{ \pm} \nu j j \bar{b} b$, it should be feasible. The $b$-jet charge can be determined from a measurement of the charges associated with the tracks in the jet. A preliminary measurement of the $b$-jet charge using Run I CDF data 60

shows a slight preference for the SM top charge assignment. The direct measurement of the $b$-jet charge has the disadvantage that many tagged $b$-quarks are needed to obtain a statistically significant result. In a given event, missing or out-of-cone tracks can distort the 
measurement. Information on the $b$-jet charge can also be obtained from the charge-sign of the lepton, $\ell_{b}=e, \mu$, in events where the $b$-quark is identified via a soft lepton tag, i.e. where the $b$-quark decays semileptonically, $b \rightarrow c \ell_{b} \nu$. In the absence of $B_{d}-\overline{B_{d}}$ and $B_{s}-\overline{B_{s}}$ mixing, the charge-sign of the lepton is directly correlated with the charge-sign of the parent $b$-quark. The difficulties associated with using soft lepton tagged $b$-quarks are low efficiency due to the small $b \rightarrow c \ell \nu$ branching ratio and the presence of wrong sign leptons originating from $B_{d}-\overline{B_{d}}$ and $B_{s}-\overline{B_{s}}$ mixing, which one has to correct for. In addition, if only the charge of the $b$ decay lepton is measured, only the sign but not the magnitude of the charge of the $b$-quark is determined.

In this paper, we explore the possibility of measuring the electric charge of the top quark at hadron colliders through photon radiation in $\bar{t} t$ events, assuming that the EM coupling strength takes its SM value'. Because top quarks can radiate photons in both top quark production and top decay, we consider both processes:

$$
p \stackrel{(-)}{p} \rightarrow \bar{t} t \gamma \quad \text { and } \quad p(\stackrel{(-)}{p} \rightarrow \bar{t} t, t \rightarrow W b \gamma
$$

We concentrate on the lepton + jets modet,

$$
p \stackrel{(-)}{p} \rightarrow \gamma \ell^{ \pm} \nu b \bar{b} j j
$$

$\ell=e, \mu$, and assume that both $b$-quarks are tagged. We present results for the SM top quark and for comparison we also consider a charge $-4 / 3$ quark of the type discussed in [4].

Photon radiation in top quark events was not observed by the Tevatron experiments in Run I. In Run II, an integrated luminosity of $2-20 \mathrm{fb}^{-1}$ is envisioned, and a sufficient number of $\gamma \ell^{ \pm} \nu b \bar{b} j j$ events may be available to measure the top quark charge. At the LHC, where the cross section for $\bar{t} t$ production is more than a factor 100 larger than at the Tevatron, one can hope for a determination of $q_{t o p}$ with a precision of about $10 \%$.

Details of our calculation are given in Sec. II. In Sec. III we present results of our numerical studies. In addition to total cross sections, distributions for the transverse momentum of the photon and the separation between the photon and the $b$-quarks in the pseudorapidity azimuthal angle plane are found to be sensitive to $q_{t o p}$. In Sec. IV we perform a quantitative analysis of how well the charge of the top quark can be measured at the Tevatron and the LHC. Finally, in Sec. V, we present our conclusions.

\section{DETAILS OF THE CALCULATION}

Our calculation is carried out at the tree level. We assume that both $b$-quarks are tagged with a combined efficiency of $40 \%$, which is included in all our numerical results. Top quark

\footnotetext{
${ }^{1}$ This is required by electromagnetic gauge invariance for on-shell photons radiated in $\bar{t} t$ events.

${ }^{2}$ Although di-lepton events, $p \stackrel{(-)}{p} \rightarrow \gamma \ell^{+} \nu \ell^{-} \bar{\nu} \bar{b} b$, are cleaner than lepton + jets events, their branching fraction is about a factor 6 smaller than that of the lepton+jets mode. The all-jets mode has a larger branching ration than lepton+jets, but is plagued by a large QCD background and, therefore, is also not considered here.
} 
and $W$ boson decays are treated in the narrow width approximation. In this approximation, there are three contributions to $p \stackrel{(-)}{p} \rightarrow \gamma \ell^{ \pm} \nu b \bar{b} j j$ :

- Radiation in top production: $p \stackrel{(-)}{p} \rightarrow \bar{t} t \gamma \rightarrow \gamma \ell^{ \pm} \nu b \bar{b} j j$.

- Radiative top decay: $p \stackrel{(-)}{p} \rightarrow \bar{t} t \rightarrow \gamma W^{+} b W^{-} \bar{b} \rightarrow \gamma \ell^{ \pm} \nu b \bar{b} j j$. Here either the $t$ or $\bar{t}$ quark decays radiatively. We denote these decays generically by $t \rightarrow W b \gamma$.

- Radiative $W$ decay: $p \stackrel{(-)}{p} \rightarrow \bar{t} t \rightarrow W^{+} b W^{-} \bar{b} \rightarrow \gamma \ell^{ \pm} \nu b \bar{b} j j$. In this case, one of the $W$ bosons decays radiatively, $W \rightarrow \ell \nu \gamma$ or $W \rightarrow j j \gamma$.

Both $\bar{t} t \gamma$ production and radiative top decays are sensitive to $q_{t o p}$. On the other hand, radiative $W$ decays are not, and should be suppressed by cuts. This can be achieved by requiring that

$$
m(j j \gamma)>90 \mathrm{GeV} \quad \text { and } \quad m_{T}\left(\ell \gamma ; \not p_{T}\right)>90 \mathrm{GeV}
$$

where $\not_{T}$ denotes the missing transverse momentum originating from the neutrino in the event which is not observed, and $m(j j \gamma)$ is the invariant mass of the $j j \gamma$ system. $m_{T}\left(\ell \gamma ; p_{T}\right)$ is the $\ell \gamma p_{T}$ cluster transverse mass, which is given by

$$
m_{T}^{2}\left(\ell \gamma ; p_{T}\right)=\left(\sqrt{p_{T}^{2}(\ell \gamma)+m^{2}(\ell \gamma)}+p_{T}\right)^{2}-\left(\mathbf{p}_{\mathbf{T}}(\ell \gamma)+\boldsymbol{p}_{\mathbf{T}}\right)^{2}
$$

where $p_{T}(\ell \gamma)$ and $m(\ell \gamma)$ are the transverse momentum and the invariant mass of the $\ell \gamma$ system, respectively. For $W \rightarrow \ell \nu \gamma$, the cluster transverse mass sharply peaks at the $W$ boson mass, $M_{W}$. In the narrow width approximation, the cuts listed in Eq. (3) completely eliminate the contributions from radiative $W$ decays to the $\gamma \ell^{ \pm} \nu b \bar{b} j j$ final state. We thus ignore radiative $W$ decays in the following.

The matrix elements for

$$
g g, \bar{q} q \rightarrow \bar{t} t \gamma \rightarrow \gamma \ell^{ \pm} \nu b \bar{b} j j
$$

are calculated using MADGRAPH [7] and the HELAS library [8], including the spin correlations for the subsequent top decays. To compute the matrix elements for

$$
g g, \bar{q} q \rightarrow \bar{t} t, \quad t \rightarrow W^{+} b \gamma, \quad \bar{t} \rightarrow W^{-} \bar{b}
$$

and its charge conjugate sibling, we use the crossed form of the $W \rightarrow t b \gamma$ matrix elements of Ref. 9]. We ignore spin correlations in the decay of the $W$ boson in $t \rightarrow W^{+} b \gamma$. For the $\bar{t} \rightarrow W^{-} \bar{b}$ decay, spin correlations are taken into account. All numerical results presented below are obtained summing over electron and muon final states.

The top quark charge, $q_{t o p}$, is treated as a free parameter in our calculation. The electric charge of the $b$-quark, $q_{b}$, is related to $q_{t o p}$ by

$$
q_{b}=q_{t o p}-q_{W}
$$

where $q_{W}= \pm 1$ is the charge of the $W$ boson. The sign of $q_{W}$ depends on whether the third component of the weak isospin of the "top quark" is $T_{3}=+1 / 2$, as in the SM, or 
$T_{3}=-1 / 2$ as in the scenario of Ref. [4]. We assume the $W t b$ coupling to be of $V-A$ form and $\operatorname{BR}(t \rightarrow W b)=1$. The fermions originating from $W$ decays are assumed to be massless.

We note for future reference that the differential cross sections for $g g \rightarrow \bar{t} t \gamma$ and for $q \bar{q}, g g \rightarrow \bar{t} t$ with one top quark decaying via $t \rightarrow W b \gamma$ are invariant under the transformation

$$
q_{t o p} \rightarrow-q_{t o p}, \quad q_{W} \rightarrow-q_{W}, \quad q_{b} \rightarrow-q_{b} .
$$

Since interference effects between the incoming light quarks and the final state top quarks in $q \bar{q} \rightarrow \bar{t} t \gamma$ are very small (they contribute less than $1 \%$ to the cross section), the cross section for $p(\vec{p}) \rightarrow \gamma \ell^{ \pm} \nu b \bar{b} j j$ is to a very good approximation invariant under the transformation (8). This will be relevant when we estimate how well $q_{t o p}$ can be measured in future Tevatron and LHC experiments (see Sec. IV).

For all our numerical simulations we have chosen the set of SM input parameters to be: $m_{t}=175 \mathrm{GeV}$ 10,11, $m_{b}=5 \mathrm{GeV}, M_{W}=80.3 \mathrm{GeV}, \sin ^{2} \theta_{W}=0.23$ and $\alpha\left(M_{W}\right)=$ $1 / 128$ [12]. We employ MRSR2 parton distribution functions [13], selecting the value of the factorization scale to be $\mu_{f}=\sqrt{\hat{s}}$, where $\hat{s}$ is the squared parton center of mass energy. Calculations are carried out for $p \bar{p}$ collisions at $\sqrt{s}=2 \mathrm{TeV}$ (Tevatron) and $p p$ collisions at $\sqrt{s}=14 \mathrm{TeV}(\mathrm{LHC})$.

To simulate detector response, we impose the following common transverse momentum, rapidity and separation cuts at the Tevatron and LHC:

$$
\begin{aligned}
p_{T}(b) & >15 \mathrm{GeV}, & & |\eta(b)|<2, \\
p_{T}(\ell) & >20 \mathrm{GeV}, & & |\eta(\ell)|<2.5, \\
p_{T}\left(j_{1,2}\right) & >20 \mathrm{GeV}, & & \left|\eta\left(j_{1,2}\right)\right|<2.5, \\
p_{T} & >20 \mathrm{GeV}, & & \Delta R(i, j)>0.4 \text { for } i \neq j,
\end{aligned}
$$

where $i, j=\gamma, \ell, b, \bar{b}, j_{1}, j_{2} . j_{1}$ and $j_{2}$ denote the two jets originating from the hadronically decaying $W$ in the event. As mentioned above, we assume that both $b$ quarks are tagged (40\% combined efficiency) so that $b$ and $\bar{b}$ are distinguishable from $j_{1}$ and $j_{2}$. In addition, we require that

$$
\begin{array}{lll}
p_{T}(\gamma)>10 \mathrm{GeV}, & |\eta(\gamma)|<2.5 & \text { at the Tevatron and } \\
p_{T}(\gamma)>30 \mathrm{GeV}, & |\eta(\gamma)|<2.5 & \text { at the LHC. }
\end{array}
$$

Here, $\eta$ is the pseudo-rapidity, and

$$
\Delta R(i, j)=\left[(\Delta \Phi(i, j))^{2}+(\Delta \eta(i, j))^{2}\right]^{1 / 2}
$$

is the separation between two particles $i$ and $j$ in the pseudorapidity - azimuthal angle plane.

The transverse momentum cuts listed in Eqs. (10) - (12) and in Eq. (14) for the LHC are sufficient for running at low luminosity, $\mathcal{L}=10^{33} \mathrm{~cm}^{-2} \mathrm{~s}^{-1}$. For operation at the design luminosity of $\mathcal{L}=10^{34} \mathrm{~cm}^{-2} \mathrm{~s}^{-1}$, the $p_{T}$ cuts must be increased due to pile-up effects caused by the large number of interactions per beam crossing. In the following we only consider $\gamma \ell^{ \pm} \nu b \bar{b} j j$ production at the LHC in the low luminosity environment.

We do not consider any potential background processes here. The dominant background is expected to originate from $W \gamma+$ jets production and should be manageable in much the same way as the $W+$ jets background for $t \bar{t}$ production. 


\section{PHENOMENOLOGICAL RESULTS}

In this section we present our results for total cross sections and various photon distributions. We look at contributions from individual subprocesses (radiative production, radiative decays) and compare results for the SM top quark to those for an exotic charge $-4 / 3$ top (as in Ref. [4]) in order to determine which quantities are sensitive to the value of $q_{\text {top }}$.

The events passing the cuts listed in Eqs. (3) and (10) - (14) can be split into three separate samples, each designed to enhance one of the three subprocesses: (i) radiation in top production (" $\bar{t} t \gamma$ selection cuts"), (ii) radiative top decay with leptonically decaying $W$ (" $t \rightarrow W b \gamma \rightarrow \ell \nu b \gamma$ selection cuts"), and (iii) radiative top decay with hadronically decaying $W($ (" $t \rightarrow W b \gamma \rightarrow j j b \gamma$ selection cuts").

The event sample for radiation in top production ( $\bar{t} t \gamma$ selection cuts) is obtained by suppressing radiative top decay events. We do this by selecting events which satisfy

$$
m\left(b_{1,2} j j \gamma\right)>190 \mathrm{GeV} \quad \text { and } \quad m_{T}\left(b_{2,1} \ell^{ \pm} \gamma ; p_{T}\right)>190 \mathrm{GeV}
$$

Here, $b_{1}, b_{2}=b, \bar{b}$ and $b_{1} \neq b_{2}$. We note that by imposing the cuts on either $b$-quark combination, we conservatively assume that the charges of the individual $b$ quarks have not been determined.

The total cross sections for $q_{t o p}=2 / 3$ and $q_{t o p}=-4 / 3$, imposing the $\bar{t} t \gamma$ selection cuts of Eq. (16) and the cuts described in Sec. II, are listed in Table Ila for the Tevatron and the LHC. At the Tevatron, the $\bar{t} t \gamma$ cross section is completely dominated by $q \bar{q}$ annihilation. As a result, photon radiation off the initial state quarks constitutes an irreducible background which limits the sensitivity of the cross section to $q_{\text {top }}$. In contrast, at the LHC more than $75 \%$ of the $\bar{t} t \gamma$ cross section originates from gluon fusion. Since gluons do not radiate photons, the LHC cross section scales approximately with $q_{t o p}^{2}$. Radiative top decays contribute about $10-20 \%(1 \%)$ to the cross section for $q_{t o p}=2 / 3\left(q_{t o p}=-4 / 3\right)$ in this phase space region. At both the Tevatron and LHC, increasing $\left|q_{t o p}\right|$ increases the total cross section with $\bar{t} t \gamma$ selection cuts.

The photon transverse momentum distributions for the individual contributions to the $\gamma \ell^{ \pm} \nu b \bar{b} j j$ differential cross section at the Tevatron (LHC) for $\bar{t} t \gamma$ selection cuts are shown in Fig. 1 (Fig. 2) for both $q_{t o p}=2 / 3$ and $q_{t o p}=-4 / 3$, keeping the same vertical scale for both charges. The cross section from $\bar{t} t$ production followed by $t \rightarrow W b \gamma \rightarrow \ell \nu b \gamma$ is about $20 \%$ larger that from $p \bar{p} \rightarrow \bar{t} t$ followed by $t \rightarrow W b \gamma \rightarrow j j b \gamma$ over most of the $p_{T}$ range considered. At the LHC, the $p_{T}(\gamma)$ distribution for the radiative top decay channels drops much faster than that for $p \stackrel{(-)}{p} \rightarrow \bar{t} t \gamma$.

A sample for radiative top decay with leptonically decaying $W(t \rightarrow W b \gamma \rightarrow \ell \nu b \gamma$ selection cuts) is obtained by requiring that

$$
m_{T}\left(b_{1,2} \ell^{ \pm} \gamma ; p_{T}\right)<190 \mathrm{GeV} \quad \text { and } \quad m\left(b_{2,1} j j \gamma\right)>190 \mathrm{GeV}
$$

in addition to (16) being not satisfied. These cuts enhance the contribution of the process

$p \stackrel{(-)}{p} \rightarrow t \bar{t}$, with $t \rightarrow W b \gamma \rightarrow \ell \nu b \gamma$. Similarly, to get events with radiative top decay and hadronically decaying $W(t \rightarrow W b \gamma \rightarrow j j b \gamma$ selection cuts) we impose

$$
m_{T}\left(b_{1,2} \ell^{ \pm} \gamma ; p_{T}\right)>190 \mathrm{GeV} \quad \text { and } \quad 160 \mathrm{GeV}<m\left(b_{2,1} j j \gamma\right)<190 \mathrm{GeV}
$$


and require that (16) is not fulfilled. We thereby obtain an event sample in which the process $p(\vec{p} \rightarrow t \bar{t}$, with $t \rightarrow W b \gamma \rightarrow j j b \gamma$ is enhanced. The cuts are imposed on either of the $b$-quark combinations, i.e. we assume that it has not been determined whether the $b$ or the $\bar{b}$ originates from the radiative top quark decay. Since the finite experimental resolution of detectors significantly broadens the Breit-Wigner resonance for $t \rightarrow W b \gamma \rightarrow j j b \gamma$, we have chosen a relatively large window for $m\left(b_{2,1} j j \gamma\right)$ around $m_{t}$. Requiring that events do not satisfy (16) significantly improves the efficiency of the $t \rightarrow W b \gamma$ selection cuts.

The cross sections for $p(\vec{p}) \rightarrow \gamma \ell^{ \pm} \nu b \bar{b} j j$ imposing $t \rightarrow W b \gamma \rightarrow \ell \nu b \gamma$ (Eq. (17)) and $t \rightarrow W b \gamma \rightarrow j j b \gamma$ selection cuts (Eq. (18)) are shown in Table \b and 【c for $q_{t o p}=2 / 3$ and $q_{\text {top }}=-4 / 3$. The total cross sections are smaller than the corresponding numbers for $\bar{t} t \gamma$ selection cuts. Moreover, the cross section for $\bar{t} t$ production followed by radiative $t$ decay decreases by a factor 3 to 4 when the top quark charge is varied from $q_{\text {top }}=2 / 3$ to $q_{\text {top }}=-4 / 3$. This is due to interference effects between the diagrams where the photon is radiated from the $t$-quark line and the diagrams where the photon is either emitted from the final state $W$ or the $b$-quark. For the photon $p_{T}$ cut used, a significant fraction of the events in the SM at the Tevatron in the regions defined by the $t \rightarrow W b \gamma \rightarrow \ell \nu b \gamma$ and $t \rightarrow W b \gamma \rightarrow j j b \gamma$ selection cuts originates from $\bar{t} t \gamma$ production. For $q_{t o p}=-4 / 3$, $\bar{t} t \gamma$ production is in fact the dominant source in these regions. Nonetheless, the net result is a decrease in the total cross section when we switch from $q_{t o p}=2 / 3$ to $q_{t o p}=-4 / 3$. Recall that, in contrast, the cross section increased with the change in top charge for $\bar{t} t \gamma$ selection cuts. This suggests that the ratio of the cross section associated with radiation in top production to the radiative decay cross section would be very sensitive to the top quark charge. This issue is explored in the next section.

Combining the cross sections for all three phase space regions, we expect about 60 $\gamma \ell^{ \pm} \nu b \bar{b} j j$ events at the Tevatron for $20 \mathrm{fb}^{-1}$. The number of events expected at the LHC is about an order of magnitude larger: $550 \gamma \ell^{ \pm} \nu b \bar{b} j j$ events for $10 \mathrm{fb}^{-1}$.

Returning to the radiative decay sample, we show in Fig. 30 the individual contributions to the $\gamma \ell^{ \pm} \nu b \bar{b} j j$ differential cross section for $t \rightarrow W b \gamma \rightarrow \ell \nu b \gamma$ selection cuts at the Tevatron for $q_{t o p}=2 / 3$ and $q_{t o p}=-4 / 3$ as a function of the photon $p_{T}$. The individual contributions at the LHC are shown in Fig. 国. We see that the contribution from $\bar{t} t \gamma$ production in the SM at the Tevatron exceeds that from $p \bar{p} \rightarrow \bar{t} t$ with $t \rightarrow W b \gamma \rightarrow \ell \nu b \gamma$ only for $p_{T}(\gamma)<25 \mathrm{GeV}$ (Fig. 3a). Increasing the photon $p_{T}$ cut thus improves the efficiency of the $t \rightarrow W b \gamma \rightarrow$ $\ell \nu b \gamma$ selection cuts, at the cost of a reduced event rate. Because the cross sections for the radiative top decay processes decrease whereas the $\bar{t} t \gamma$ rate increases when $q_{t o p}=-4 / 3, \bar{t} t \gamma$ production is the largest contribution to the differential cross section over the entire $p_{T}(\gamma)$ range considered at both the Tevatron and the LHC (Figs. 33b and 해 $\mathrm{b}$. The differential cross section of the $t \rightarrow W b \gamma \rightarrow j j b \gamma$ "background" drops much faster with $p_{T}(\gamma)$ than those of the two other contributions for large photon transverse momenta. When $t \rightarrow W b \gamma \rightarrow$ $j j b \gamma$ selection cuts are imposed, qualitatively similar results are obtained, with the roles of the $t \rightarrow W b \gamma \rightarrow \ell \nu b \gamma$ and $t \rightarrow W b \gamma \rightarrow j j b \gamma$ curves exchanged and somewhat reduced contributions from $\bar{t} \gamma$ production.

The preceding results are summarized in Figs. 5 and 6. Fig. 5a shows the total SM $\left(q_{\text {top }}=+2 / 3\right)$ cross section for $p \bar{p} \rightarrow \gamma \ell^{ \pm} \nu b \bar{b} j j$ at the Tevatron, with all contributions from radiation in top production and radiative decays combined. The three curves correspond to the three sets of selection cuts. Fig. 6a shows the corresponding results for the LHC (with 
$p p$ initial state). We see in these figures, as we have already seen in Table [ , that the $\bar{t} t \gamma$ selection cuts (solid curves) result in higher cross sections at both colliders than the cuts for radiative decays $t \rightarrow W b \gamma$. In Figs. 5a and 6a we also see that the photon distribution imposing $t \rightarrow W b \gamma \rightarrow \ell \nu b \gamma$ and $t \rightarrow W b \gamma \rightarrow j j b \gamma$ selection cuts drops much faster than the $p_{T}(\gamma)$ distribution in the phase space region defined by the $\bar{t} t \gamma$ selection cuts. This is a consequence of the phase space restriction on the photon energy in radiative top quark decays.

In Figs. 5b and 6 $\mathrm{b}$ we compare cross sections for $q_{t o p}=+2 / 3$ (solid curves) and $q_{t o p}=$ $-4 / 3$ (dashed curves) for the Tevatron and LHC, respectively. In each figure the upper pair of curves corresponds to $\bar{t} t \gamma$ selection cuts and the lower pair includes events from both radiative decay regions combined. The figures show that at both machines, the effect of increasing $\left|q_{t o p}\right|$ is to increase the cross section in the $\bar{t} t \gamma$ cuts region and to decrease it in the radiative decay regions, for all values of photon $p_{T}$. At the Tevatron, however, since the contributions from radiative top quark decays are small if $\bar{t} t \gamma$ selection cuts are imposed, changing the top quark charge has little influence on the shape of the photon $p_{T}$ distribution in this region (upper curves in Fig. 5b). On the other hand, at small values of $p_{T}(\gamma), \bar{t} t \gamma$ production may contribute significantly to the cross section in the regions defined by the $t \rightarrow W b \gamma \rightarrow \ell \nu b \gamma$ and $t \rightarrow W b \gamma \rightarrow j j b \gamma$ selection cuts (see Table I). The shape of the photon transverse momentum distribution in these regions thus may be sensitive to $q_{\text {top }}$ (lower curves in Fig. 5b). The curves at the LHC (Fig. 66) also exhibit some shape dependence, albeit less pronounced than at the Tevatron.

We can look for sensitivity to $q_{t o p}$ in other distributions as well. In $t \rightarrow W b \gamma$, emission of the photon from the $b$-quark line leads to a collinear enhancement at small values of the separation between the photon and the $b$-quark, $\Delta R(\gamma, b)_{\text {rad }}$, which depends on $q_{b}=q_{\text {top }}-q_{W}$. Therefore, in radiative top decays, the shape of the $\Delta R(\gamma, b)_{\text {rad }}$ distribution is expected to be sensitive to the charge of the top quark. In contrast, the photon and the $b$-quark originating from the $t \rightarrow W b$ decay in $\bar{t} t \rightarrow \gamma W^{+} b W^{-} \bar{b}$ events are essentially uncorrelated. In $\bar{t} t \gamma$ events, the photon and both $b$-quarks are largely uncorrelated. Unfortunately, on an event by event basis, it is difficult to determine whether the $b$ or the $\bar{b}$ quark originates from the radiative top decay. However, due to the collinear enhancement for small values of $\Delta R$ in radiative top decays,

$$
\Delta R(\gamma, b)_{\min }=\min [\Delta R(\gamma, b), \Delta R(\gamma, \bar{b})]
$$

should coincide with the photon - $b$-quark separation in radiative top decays for small values of $\Delta R$. Alternatively, the $\Delta R\left(\gamma, b_{s f}\right)$ distribution, where $b_{s f}$ is the $b$ or $\bar{b}$-quark with the smaller transverse momentum

$$
p_{T}\left(b_{s f}\right)=\min \left[p_{T}(b), p_{T}(\bar{b})\right]
$$

may be considered. Since a $b$-quark which radiates a photon looses energy, the $b_{s f}$ in $\bar{t} t \rightarrow \gamma W^{+} b W^{-} \bar{b}$ frequently originates from the radiative top decay. It turns out that the $\Delta R\left(\gamma, b_{s f}\right)$ distribution more closely resembles the true $\Delta R(\gamma, b)_{\text {rad }}$ distribution. Figure 7 shows a comparison of the $\Delta R(\gamma, b)_{\text {rad }}$ and $\Delta R\left(\gamma, b_{s f}\right)$ distributions at the Tevatron in the SM case, imposing $t \rightarrow W b \gamma \rightarrow \ell \nu b \gamma$ selection cuts. In each case, the individual distributions of the three contributing subprocesses are shown. Qualitatively similar results are obtained for the LHC case, and for $t \rightarrow W b \gamma \rightarrow j j b \gamma$ selection cuts. Due to the collinear enhancement 
at small opening angles between the $b$-quark and the photon, the $\Delta R(\gamma, b)_{\text {rad }}$ distribution rises very quickly with decreasing values of $\Delta R(\gamma, b)_{\text {rad }}$ for the radiative top decay processes. For $\bar{t} t \gamma$ production no collinear enhancement occurs for either the $\Delta R(\gamma, b)$ or the $\Delta R(\gamma, \bar{b})$ distribution. In Fig. $7 \mathrm{a}$, we have chosen the $\Delta R(\gamma, \bar{b})$ distribution for $\bar{t} t \gamma$ production (dotted line). Very similar results are obtained if the $\Delta R(\gamma, b)$ distribution is chosen instead. Except for the steepness of the distribution at small $\Delta R\left(\gamma, b_{s f}\right)$ for $t \rightarrow W b \gamma \rightarrow \ell \nu b \gamma$, the shapes of the $\Delta R(\gamma, b)_{\text {rad }}$ and $\Delta R\left(\gamma, b_{s f}\right)$ distributions are found to agree reasonably well.

In Fig. 7 we have increased the minimum photon transverse momentum requirement of Eq. (13) to $p_{T}(\gamma)>15 \mathrm{GeV}$. As we have mentioned before, $\bar{t} t \gamma$ production contributes significantly to the cross section at the Tevatron in the region defined by the $t \rightarrow W b \gamma \rightarrow$ $\ell \nu b \gamma$ selection cuts for the cuts specified in Sec. II. The $\bar{t} t \gamma$ contribution can be reduced by increasing the $p_{T}$ cut on the photon (see Fig. 3), at the cost of a decreased number of events. Increasing the photon transverse momentum cut to $p_{T}(\gamma)>15 \mathrm{GeV}$ reduces the $\bar{t} t \gamma$ cross section for $t \rightarrow W b \gamma \rightarrow \ell \nu b \gamma$ selection cuts by about a factor 2 , whereas the contributions involving radiative top decays decrease by only $30 \%$.

We compare the $\Delta R$ distributions for the two top quark charges in Figures 8 and 9 . We display the normalized distribution $(1 / \sigma)\left(d \sigma / d \Delta R\left(\gamma, b_{s f}\right)\right)$ for $\bar{t} t \rightarrow \gamma \ell \nu j j \bar{b} b$ with $q_{t o p}=2 / 3$ and $q_{t o p}=-4 / 3$ at the Tevatron and LHC, respectively. Normalizing the distributions to unit area emphasizes the dependence of the shape of the $\Delta R\left(\gamma, b_{s f}\right)$ distribution to the top quark charge. For a charge $-4 / 3$ quark, the $\Delta R\left(\gamma, b_{s f}\right)$ distribution rises relatively faster in the region $\Delta R\left(\gamma, b_{s f}\right) \leq 1$, but is flatter for $1<\Delta R\left(\gamma, b_{s f}\right)<3$, than if $q_{\text {top }}=2 / 3$. At small values of $\Delta R\left(\gamma, b_{s f}\right)$, emission from the $b$-quark line dominates. Since the magnitude of $q_{b}$ is the same for $q_{t o p}=2 / 3$ and $q_{t o p}=-4 / 3$, the differential cross section is similar in both cases. However, the total cross section for $q_{t o p}=-4 / 3$ within cuts is significantly smaller than for a SM top quark charge assignment (see Table $\mathbb{\text { Q) }}$ ), due to destructive interference between the contributing Feynman diagrams. This leads to a larger normalized differential cross section for $q_{t o p}=-4 / 3$ at small $\Delta R\left(\gamma, b_{s f}\right)$. In the region $1<\Delta R\left(\gamma, b_{s f}\right)<3$, the destructive interference is responsible for the flatness of the $(1 / \sigma)\left(d \sigma / d \Delta R\left(\gamma, b_{s f}\right)\right)$ distribution in the $q_{\text {top }}=-4 / 3$ case. For $\Delta R\left(\gamma, b_{s f}\right)>3$, the $b$-quark with the smaller $p_{T}$ most of the time coincides with the bottom quark from the (regular) $t \rightarrow W b$ decay and, thus, carries little information on $q_{t o p}$. The shape of the $\Delta R\left(\gamma, b_{s f}\right)$ distribution in the phase space region defined by the $t \rightarrow W b \gamma \rightarrow j j b \gamma$ selection cuts (Figs. 8b and 9b) is found to be somewhat more sensitive to $q_{\text {top }}$ than in the case when $t \rightarrow W b \gamma \rightarrow \ell \nu b \gamma$ selection cuts are imposed (Figs. 8a and 9a). At the Tevatron (Fig. 8), we have again imposed a $p_{T}(\gamma)>15 \mathrm{GeV}$ cut. The shape of the $\Delta R\left(\gamma, b_{s f}\right)$ distribution for $\bar{t} t \rightarrow \gamma W^{+} b W^{-} \bar{b}$ at the LHC is in general more sensitive to the top quark charge than at the Tevatron.

\section{QUANTITATIVE ESTIMATES}

In this section we determine quantitative bounds on

$$
\Delta q_{t o p}=q_{t o p}-\frac{2}{3}
$$

which one expects to achieve with $20 \mathrm{fb}^{-1}$ of data at the Tevatron in Run II, and $10 \mathrm{fb}^{-1}$ at the LHC, analyzing the photon $p_{T}$ and the $\Delta R\left(\gamma, b_{s f}\right)$ distributions. We also briefly consider 
the ratio of the cross section associated with radiation in top production to the radiative decay cross section which may be useful for small data sets.

In our analysis of differential cross sections, we calculate 95\% confidence level (C.L.) limits performing a $\chi^{2}$ test. The statistical significance is calculated by splitting the selected distribution into a number of bins, each with typically more than five events. In each bin the Poisson statistics are approximated by a Gaussian distribution. We use the photon transverse momentum distribution in the phase space region defined by the $\bar{t} t \gamma$ selection cuts of (16), and the $p_{T}(\gamma)$ and $\Delta R\left(\gamma, b_{s f}\right)$ distributions of the combined radiative top decay regions, defined by requiring that events satisfy (17) or (18) but not (16). We impose the cuts described in Sec. II, except for the $\Delta R\left(\gamma, b_{s f}\right)$ distribution, where we replace the $p_{T}$ cut on the photon of Eq. (13) with $p_{T}(\gamma)>15 \mathrm{GeV}$. We combine channels with electrons and muons (both charge combinations) in the final state. We also make use of the approximate symmetry of the cross sections under the transformation (8) and define the $b$-quark charge to be $q_{b}=q_{t o p}-1$. Confidence levels calculated for $q_{t o p}=+4 / 3$ will thus automatically apply for a charge $-4 / 3$ quark which decays into a $b$-quark and a $W^{-}$.

Our calculation does not include QCD corrections which are expected to modify the cross section by $20-40 \%$. In order to derive realistic limits, we therefore allow for a normalization uncertainty of $\Delta \mathcal{N}=30 \%$ of the SM cross section. The expression for $\chi^{2}$ which is then used to compute confidence levels is given by [14]

$$
\chi^{2}=\sum_{i=1}^{n_{D}} \frac{\left(N_{i}-f N_{i}^{0}\right)^{2}}{f N_{i}^{0}}+\left(n_{D}-1\right),
$$

where $n_{D}$ is the number of bins, $N_{i}$ is the number of events for a given $\Delta q_{t o p}$, and $N_{i}^{0}$ is the number of events in the SM in the $i$ th bin. $f$ reflects the uncertainty in the normalization of the SM cross section within the allowed range, and is determined by minimizing $\chi^{2}$ :

$$
f= \begin{cases}(1+\Delta \mathcal{N})^{-1} & \text { for } \bar{f}<(1+\Delta \mathcal{N})^{-1} \\ \bar{f} & \text { for }(1+\Delta \mathcal{N})^{-1}<\bar{f}<1+\Delta \mathcal{N} \\ 1+\Delta \mathcal{N} & \text { for } \bar{f}>1+\Delta \mathcal{N}\end{cases}
$$

with

$$
\bar{f}^{2}=\left\{\sum_{i=1}^{n_{D}} N_{i}^{0}\right\}^{-1} \sum_{i=1}^{n_{D}} \frac{N_{i}^{2}}{N_{i}^{0}}
$$

In Table 【I we display the resulting 95\% confidence level limits for $\Delta q_{\text {top }}$ expected from the Tevatron Run II for an integrated luminosity of $20 \mathrm{fb}^{-1}$, and from the LHC for $10 \mathrm{fb}^{-1}$. The last row shows the combined limits from the three individual distributions studied here. Possible backgrounds are assumed to be subtracted. At both the Tevatron and the LHC, the $p_{T}(\gamma)$ and $\Delta R\left(\gamma, b_{s f}\right)$ distributions in the combined radiative top decay regions yield rather similar limits for $\Delta q_{t o p}$. As mentioned before, $\bar{t} t \gamma$ production at the Tevatron is completely dominated by $q \bar{q}$ annihilation and photon radiation from the initial state quarks limits the sensitivity of the cross section to $q_{t o p}$. Therefore, the bounds which can be achieved at the Tevatron from analyzing the photon $p_{T}$ distribution in the phase space region defined by the $\bar{t} t \gamma$ selection cuts are significantly weaker than those which can be obtained from either 
the $p_{T}(\gamma)$ or the $\Delta R\left(\gamma, b_{s f}\right)$ distribution in the combined radiative top decay regions. [5 At the LHC, most of the $\bar{t} t \gamma$ cross section originates from gluon fusion, and the limits from the photon $p_{T}$ distribution for $\bar{t} t \gamma$ selection cuts are similar to those which can be achieved in the phase space regions where radiative top decays dominate.

While a precise measurement of the top quark charge will not be possible in Run II of the Tevatron, even with an integrated luminosity of $20 \mathrm{fb}^{-1}$, we note that a charge $-4 / 3$ top quark can be ruled out at $\approx 95 \%$ C.L. when the limits from the different distributions and phase space regions are combined. At the LHC, with $10 \mathrm{fb}^{-1}$, a measurement of the top quark charge with an accuracy of about $10 \%$ appears to be feasible. As we pointed out in Sec. II, the $p_{T}$ cuts we use are suitable for running at low luminosity at the LHC, i.e. for $\mathcal{L}=10^{33} \mathrm{~cm}^{-2} \mathrm{~s}^{-1}$. At high luminosity, $\mathcal{L}=10^{34} \mathrm{~cm}^{-2} \mathrm{~s}^{-1}$, the transverse momentum cuts must be increased due to pile-up effects caused by the large number of interactions per beam crossing. The $\gamma \ell^{ \pm} \nu b \bar{b} j j$ cross section is quite sensitive to these cuts. For example, increasing the $p_{T}$ cut of the $b$-quarks to $30 \mathrm{GeV}$ and the photon, jet and missing $p_{T}$ cuts to $50 \mathrm{GeV}$ reduces the $\bar{t} t \gamma$ and $\bar{t} t \rightarrow \gamma W^{+} b W^{-} \bar{b}$ cross sections by a factor $\approx 10$ and $\approx 100$, respectively. It is thus not clear whether running at high luminosity will improve the measurement of the top quark electric charge at the LHC.

The bounds on $\Delta q_{\text {top }}$ are in general quite sensitive to $\Delta \mathcal{N}$. If the normalization uncertainty can be reduced to $\Delta \mathcal{N}=10 \%$, the limits listed in Table $\mathbb{I}$ can be improved by up to a factor 2. Further improvements may result from using more powerful statistical tools than the simple $\chi^{2}$ test we performed.

For integrated luminosities smaller than $20 \mathrm{fb}^{-1}$ at the Tevatron, the limited number of events makes a detailed analysis of differential cross sections difficult. In this case, the ratio of the cross section associated with radiation in top production to the radiative decay cross section, $\mathcal{R}$, may be more useful. Many experimental and theoretical uncertainties, for example, those associated with the particle detection efficiencies, the uncertainty in the integrated luminosity, or the uncertainties from the choice of scale or of the parton distribution functions, are expected to cancel, at least partially, in $\mathcal{R}$. The cross section ratio is quite sensitive to $q_{\text {top }}$. From the individual cross sections listed in Table \ one finds $\mathcal{R}=1.73$ for $q_{\text {top }}=2 / 3$, and $\mathcal{R}=2.88$ for $q_{\text {top }}=-4 / 3$ at the Tevatron. For an integrated luminosity of $10 \mathrm{fb}^{-1}$, the expected statistical uncertainty at the Tevatron is $\Delta \mathcal{R}_{\text {stat }}=0.65$ and a charge $-4 / 3$ top quark can thus be ruled out at $\approx 90 \%$ C.L. using the cross section ratio, if one ignores possible systematic uncertainties. At the LHC, the value of $\mathcal{R}$ is even more sensitive to $q_{t o p}$ than at the Tevatron, but enough events are expected to allow a more detailed analysis.

\section{SUMMARY AND CONCLUSIONS}

Like most of its fundamental quantum numbers, the electric charge of the top quark has not been measured so far. Alternative interpretations for the particle we believe is the

\footnotetext{
${ }^{3}$ In practice, hadronization effects we have omitted will come into play, and are likely to affect the $\Delta R$ distributions more than those for the photon $p_{T}$.
} 
charge $2 / 3$ isospin partner of the $b$-quark are thus not ruled out. For example, since the correlations of the $b$-quarks and the $W$-bosons in $\bar{p} p \rightarrow \bar{t} t \rightarrow W^{+} W^{-} \bar{b} b$ are not determined, it is conceivable that the "$t$-quark" observed at the Tevatron is an exotic quark, $Q_{4}$, with charge $-4 / 3$ which decays via $Q_{4} \rightarrow W^{-} b$. This interpretation is consistent with current precision electroweak data [四]. In order to determine the charge of the top quark, one can either measure the charge of the $b$-jet, or investigate photon radiation in $\bar{t} t$ events. The latter method actually measures a combination of the EM coupling strength and the charge quantum number. Combining the results of the two methods will thus make it possible to determine both quantities.

In this paper we have explored the possibility of measuring $q_{t o p}$ in $p\left(\vec{p} \rightarrow \gamma \ell^{ \pm} \nu \bar{b} b j j\right.$, assuming that both $b$-quarks are tagged. Our analysis makes use of both $\bar{t} t \gamma$ production and radiative top quark decays, and is carried out at the tree level. The EM coupling strength is assumed to take its SM value. Top quark and $W$ boson decays are treated in the narrow width approximation. Contributions from radiative $W$ decays can be suppressed by simple phase space cuts (see Eq. (3)). The remaining event sample can be separated into a $\bar{t} t \gamma$ sample, a $\bar{t} t, t \rightarrow W b \gamma \rightarrow \gamma \ell \nu b$ sample, and a $\bar{t} t, t \rightarrow W b \gamma \rightarrow \gamma j j b$ sample by imposing invariant mass and cluster transverse mass cuts (see Eqs. (16) - (18)).

In the phase space region dominated by $\bar{t} t \gamma$ production, the cross section increases if the magnitude of $q_{t o p}$ increases. At the Tevatron, $q \bar{q}$ annihilation dominates and photon radiation off the incoming quarks constitutes an irreducible background which limits the sensitivity to $q_{t o p}$. In contrast, at the LHC, gluon fusion dominates, and the $\bar{t} t \gamma$ cross section scales approximately with $q_{\text {top }}^{2}$.

In the phase space regions which enhance $\bar{t} t$ production with one of the top quarks decaying radiatively $(t \rightarrow W b \gamma)$, interference effects between the Feynman diagrams where the photon is emitted from the top quark, the $W$ boson and the $b$-quark line may cause the cross section to either decrease or increase with $\Delta q_{t o p}=q_{t o p}-2 / 3$. For example, for $q_{\text {top }}=-4 / 3$, the $\gamma \ell^{ \pm} \nu \bar{b} b j j$ cross section via radiative top quark decays is reduced by a factor of 3 to 4 .

The changes in the individual cross sections are reflected in the photon transverse momentum distribution in the various phase space regions. We also found that the $\Delta R\left(\gamma, b_{s f}\right)$ distribution, where $b_{s f}$ is the $b$ or $\bar{b}$-quark with the smaller $p_{T}$, is sensitive to the charge of the top quark in the phase space regions dominated by $\bar{t} t$ production where one of the top quarks decays radiatively.

In order to determine how well one can hope to measure the top quark charge using photon radiation in top quark events in future Tevatron and LHC experiments, we have performed $\chi^{2}$ tests of the $p_{T}(\gamma)$ and the $\Delta R\left(\gamma, b_{s f}\right)$ distributions in the various phase space regions of interest. At the Tevatron, with an integrated luminosity of $20 \mathrm{fb}^{-1}$, one will be able to exclude at $\approx 95 \%$ C.L. the possibility that an exotic quark $Q_{4}$ with charge $-4 / 3$ and not the SM top quark has been found in Run I. For smaller integrated luminosities, the number of events expected is very small and it will be difficult to perform a quantitative analysis using differential cross sections. In this case, the ratio of the cross section associated with radiation in top production to the radiative decay cross section may be a useful tool. At the LHC with $10 \mathrm{fb}^{-1}$ obtained at $\mathcal{L}=10^{33} \mathrm{~cm}^{-2} \mathrm{~s}^{-1}$, it should be possible to measure the electric charge of the top quark with an accuracy of about 10\%. For comparison, at a linear collider with $\sqrt{s}=500 \mathrm{GeV}$ and $\int \mathcal{L} d t=200 \mathrm{fb}^{-1}$, one expects that $q_{\text {top }}$ can also be 
measured with a precision of about $10 \%$ 15]. Finally, at a $\gamma \gamma$ collider it is conceivable that the top quark charge can be determined with an accuracy of better than $1 \%$, if the $\gamma \gamma \rightarrow \bar{t} t$ cross section can be measured with a precision of $2 \%$ [16].

The measurement of the electric charge of the top quark at the Tevatron using photon radiation in top quark events is severely limited by statistics. Even with an integrated luminosity of $20 \mathrm{fb}^{-1}, q_{\text {top }}$ can only be determined with a precision of $30-100 \%$. However, as we pointed out in Sec. I, information on the electric charge of the top quark may also be obtained from a measurement of the charge of the $b$-jets, and the charge sign of the leptons in semileptonically tagged $b$-quarks. This may significantly improve the precision which can

be obtained. Detailed simulations will be necessary in order to determine how well the top quark charge can be measured using these methods.

\section{ACKNOWLEDGMENTS}

We would like to thank C. Ferreti, H. Frisch, B. Knuteson, T. LeCompte, J. Parsons, Z. Sullivan and J. Womersley for stimulating discussions. One of us (U.B.) is grateful to the Fermilab Theory Group, where part of this work was carried out, for its generous hospitality. This work has been supported in part by DOE grant DE-FG02-91ER40685, NSF grants PHY-9600155 and PHY-9970703, and a NSF Graduate Student Fellowship. 


\section{REFERENCES}

[1] F. Abe et al. (CDF Collaboration), Phys. Rev. Lett. 74, 2626 (1995).

[2] S. Abachi et al. (DØ Collaboration), Phys. Rev. Lett. 74, 2632 (1995).

[3] P. C. Bhat, H. Prosper and S. S. Snyder, Int. J. Mod. Phys. A13, 5113 (1998).

[4] D. Chang, W.F. Chang, and E. Ma, Phys. Rev. D59, 091503 (1999) and Phys. Rev. D61, 037301 (2000).

[5] B. Grzadkowski and Z. Hioki, Nucl. Phys. B585, 3 (2000) and references therein.

[6] G. Velev, talk given at the Top Thinkshop ${ }^{2}$, Fermilab, November 2000.

[7] T. Stelzer and W. F. Long, Comput. Phys. Commun. 81, 357 (1994).

[8] H. Murayama, I. Watanabe and K. Hagiwara, KEK-91-11 (1991).

[9] A. Denner and T. Sack, Z. Phys. C46, 653 (1990).

[10] T. Affolder et al., (CDF Collaboration), Phys. Rev. D63, 032003 (2001).

[11] B. Abbott et al. (DØ Collaboration), Phys. Rev. D58, 052001 (1998).

[12] D. Abbaneo et al. (The LEP Electroweak Working Group), CERN-EP-2001-021 (February 2001); hep-ex/0103048.

[13] A. D. Martin, R. G. Roberts and W. J. Stirling, Phys. Lett. B387, 419 (1996).

[14] U. Baur and E. L. Berger, Phys. Rev. D47, 4889 (1993).

[15] T. Abe et al. (American Linear Collider Working Group Collaboration), SLAC-570 Resource book for Snowmass 2001, 30 Jun - 21 Jul 2001, Snowmass, Colorado, hepex/0106055, hep-ex/0106056, hep-ex/0106057 and hep-ex/0106058.

[16] E. Boos, hep-ph/0009100, and references therein. 


\section{TABLES}

TABLE I. Integrated cross sections for $\gamma \ell^{ \pm} \nu \bar{b} b j j$ production at the Tevatron $(p \bar{p}$ collisions at $\sqrt{s}=2 \mathrm{TeV})$ and the LHC $(p p$ collisions at $\sqrt{s}=14 \mathrm{TeV})$ for $q_{\text {top }}=2 / 3$ and $q_{\text {top }}=-4 / 3$ imposing a) $\bar{t} t \gamma$ selection cuts, b) $t \rightarrow W b \gamma \rightarrow \ell \nu b \gamma$ selection cuts, and c) $t \rightarrow W b \gamma \rightarrow j j b \gamma$ selection cuts. In addition, the cuts described in Sec. II are imposed. In each case, the contributions from $\bar{t} t \gamma$ production, $\bar{t} t$ production with $t \rightarrow W b \gamma \rightarrow \ell \nu b \gamma$, and $\bar{t} t$ production with $t \rightarrow W b \gamma \rightarrow j j b \gamma$ are shown.

\begin{tabular}{lcccc}
\hline \hline \multicolumn{5}{c}{ a) $\bar{t} t \gamma$ selection cuts } \\
\hline & $\begin{array}{c}\bar{t} t \gamma \\
\text { contribution }\end{array}$ & $\begin{array}{c}t \rightarrow W b \gamma \rightarrow \ell \nu b \gamma \\
\text { contribution }\end{array}$ & $\begin{array}{c}t \rightarrow W b \gamma \rightarrow j j b \gamma \\
\text { contribution }\end{array}$ & total \\
\hline Tevatron, $q_{\text {top }}=+\frac{2}{3}$ & $1.70 \mathrm{fb}$ & $0.12 \mathrm{fb}$ & $0.10 \mathrm{fb}$ & $1.92 \mathrm{fb}$ \\
Tevatron, $q_{\text {top }}=-\frac{4}{3}$ & $2.37 \mathrm{fb}$ & $0.03 \mathrm{fb}$ & $0.02 \mathrm{fb}$ & $2.42 \mathrm{fb}$ \\
\hline LHC, $q_{\text {top }}=+\frac{2}{3}$ & $33.3 \mathrm{fb}$ & $5.78 \mathrm{fb}$ & $4.86 \mathrm{fb}$ & $44.0 \mathrm{fb}$ \\
LHC, $q_{\text {top }}=-\frac{4}{3}$ & $111.4 \mathrm{fb}$ & $1.21 \mathrm{fb}$ & $0.97 \mathrm{fb}$ & $113.6 \mathrm{fb}$ \\
\hline \multicolumn{5}{c}{$\mathrm{b}) t \rightarrow W b \gamma \rightarrow \ell \nu \gamma \gamma$ selection cuts } \\
\hline Tevatron, $q_{\text {top }}=+\frac{2}{3}$ & $0.36 \mathrm{fb}$ & $0.26 \mathrm{fb}$ & $0.13 \mathrm{fb}$ & $0.75 \mathrm{fb}$ \\
Tevatron, $q_{\text {top }}=-\frac{4}{3}$ & $0.52 \mathrm{fb}$ & $0.07 \mathrm{fb}$ & $0.03 \mathrm{fb}$ & $0.62 \mathrm{fb}$ \\
\hline LHC, $q_{\text {top }}=+\frac{2}{3}$ & $0.68 \mathrm{fb}$ & $3.67 \mathrm{fb}$ & $2.26 \mathrm{fb}$ & $6.61 \mathrm{fb}$ \\
LHC, $q_{\text {top }}=-\frac{4}{3}$ & $2.25 \mathrm{fb}$ & $0.87 \mathrm{fb}$ & $0.59 \mathrm{fb}$ & $3.71 \mathrm{fb}$ \\
\hline \multicolumn{5}{c}{$\mathrm{c} t \rightarrow W b \gamma \rightarrow j j b \gamma \mathrm{selection} \mathrm{cuts}$} \\
Tevatron, $q_{\text {top }}=+\frac{2}{3}$ & $0.11 \mathrm{fb}$ & $0.06 \mathrm{fb}$ & $0.19 \mathrm{fb}$ & $0.36 \mathrm{fb}$ \\
Tevatron, $q_{\text {top }}=-\frac{4}{3}$ & $0.16 \mathrm{fb}$ & $0.01 \mathrm{fb}$ & $0.05 \mathrm{fb}$ & $0.22 \mathrm{fb}$ \\
\hline LHC, $q_{\text {top }}=+\frac{2}{3}$ & $0.15 \mathrm{fb}$ & $1.07 \mathrm{fb}$ & $3.63 \mathrm{fb}$ & $4.85 \mathrm{fb}$ \\
LHC, $q_{\text {top }}=-\frac{4}{3}$ & $0.49 \mathrm{fb}$ & $0.26 \mathrm{fb}$ & $0.85 \mathrm{fb}$ & $1.60 \mathrm{fb}$ \\
\hline \hline
\end{tabular}

TABLE II. Limits achievable at $95 \%$ C.L. for $\Delta q_{t o p}$ in $p \stackrel{(-)}{p} \rightarrow \gamma \ell^{ \pm} \nu \bar{b} b j j$ at the Tevatron and the LHC. Bounds are shown for the photon transverse momentum distribution in the phase space region defined by the $\bar{t} t \gamma$ selection cuts, and the $p_{T}(\gamma)$ and $\Delta R\left(\gamma, b_{s f}\right)$ distributions of the combined radiative top decay regions. The last row displays the combined limits from the three distributions. The $b$-quark charge is given by $q_{b}=q_{t o p}-1$. The cuts imposed are described in Sec. II. For the $\Delta R\left(\gamma, b_{s f}\right)$ distribution, the $p_{T}(\gamma)$ cut of Eq. (13) has been replaced by $p_{T}(\gamma)>15 \mathrm{GeV}$.

\begin{tabular}{ccc}
\hline \hline distribution & Tevatron, $\int \mathcal{L} d t=20 \mathrm{fb}^{-1}$ & LHC, $\int \mathcal{L} d t=10 \mathrm{fb}^{-1}$ \\
\hline$p_{T}(\gamma), \bar{t} t \gamma$ region & $-1.16 \leq \Delta q_{\text {top }} \leq 1.11$ & $-0.21 \leq \Delta q_{\text {top }} \leq 0.24$ \\
$p_{T}(\gamma)$, comb. $t \rightarrow W b \gamma$ regions & $-0.32 \leq \Delta q_{\text {top }} \leq 1.20$ & $-0.16 \leq \Delta q_{\text {top }} \leq 0.18$ \\
$\Delta R\left(\gamma, b_{s f}\right)$, comb. $t \rightarrow W b \gamma$ regions & $-0.29 \leq \Delta q_{\text {top }} \leq 1.10$ & $-0.15 \leq \Delta q_{\text {top }} \leq 0.18$ \\
\hline combined & $-0.21 \leq \Delta q_{\text {top }} \leq 0.65$ & $-0.067 \leq \Delta q_{\text {top }} \leq 0.070$ \\
\hline \hline
\end{tabular}




\section{FIGURES}
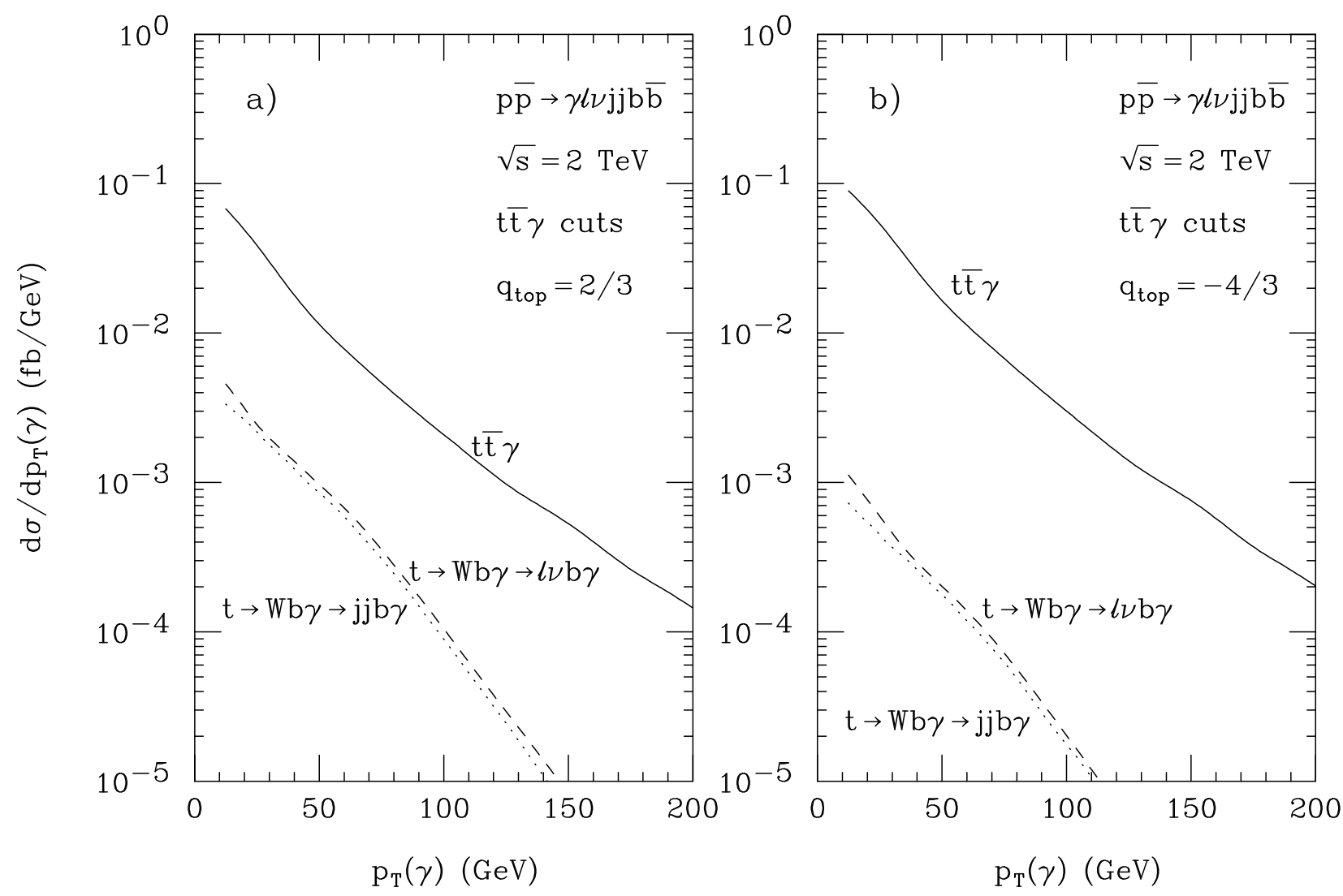

FIG. 1. The photon transverse momentum distribution for $p \bar{p} \rightarrow \gamma \ell^{ \pm} \nu b \bar{b} j j$ at the Tevatron, imposing $\bar{t} t \gamma$ selection cuts (see Eq. (16)), for a) $q_{t o p}=2 / 3$ and b) $q_{t o p}=-4 / 3$. Shown are the $\bar{t} t \gamma$ (solid line), $\bar{t} t, t \rightarrow W b \gamma \rightarrow \ell \nu b \gamma$ (dashed line), and $\bar{t} t, t \rightarrow W b \gamma \rightarrow j j b \gamma$ (dotted line) contributions. The additional cuts imposed are described in Sec. II. 

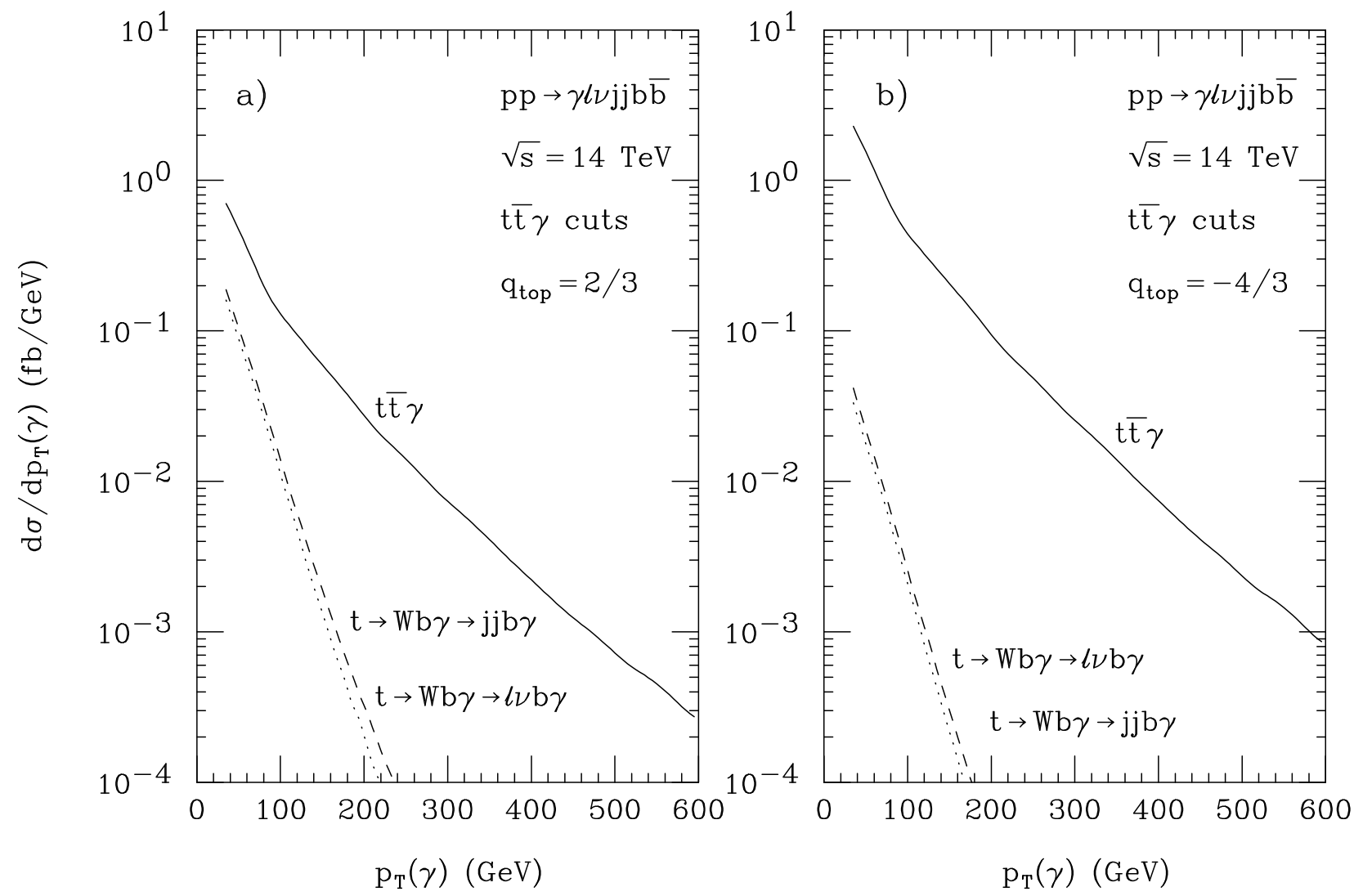

FIG. 2. The photon transverse momentum distribution for $p p \rightarrow \gamma \ell^{ \pm} \nu b \bar{b} j j$ at the LHC, imposing $\bar{t} t \gamma$ selection cuts (see Eq. (16)), for a) $q_{\text {top }}=2 / 3$ and b) $q_{t o p}=-4 / 3$. Shown are the $\bar{t} t \gamma$ (solid line), $\bar{t} t, t \rightarrow W b \gamma \rightarrow \ell \nu b \gamma$ (dashed line), and $\bar{t} t, t \rightarrow W b \gamma \rightarrow j j b \gamma$ (dotted line) contributions. The additional cuts imposed are described in Sec. II. 

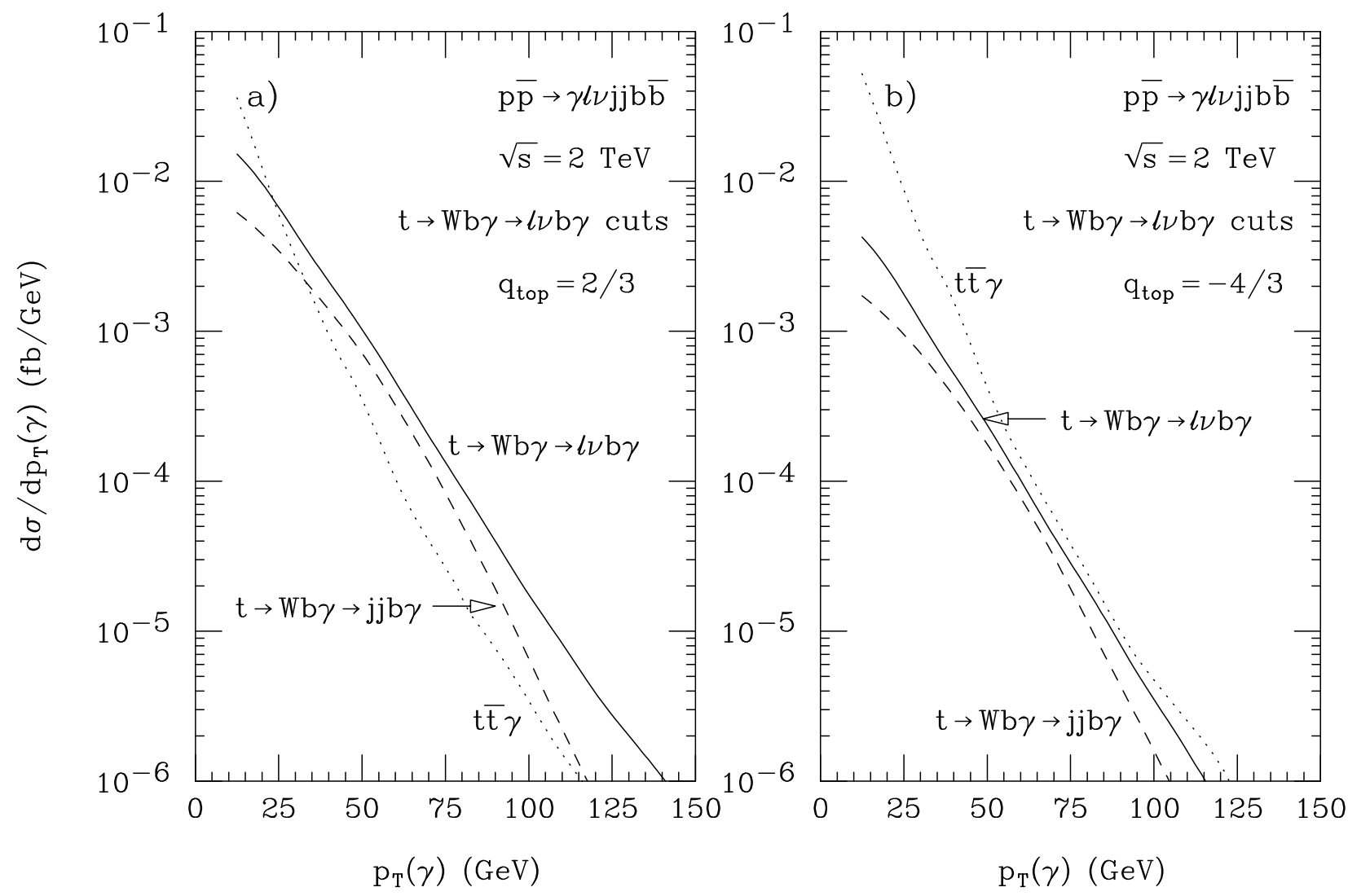

FIG. 3. The photon transverse momentum distribution for $p \bar{p} \rightarrow \gamma \ell^{ \pm} \nu b \bar{b} j j$ at the Tevatron, imposing $t \rightarrow W b \gamma \rightarrow \ell \nu b \gamma$ selection cuts (see text), for a) $q_{\text {top }}=2 / 3$ and b) $q_{\text {top }}=-4 / 3$. Shown are the $\bar{t} t, t \rightarrow W b \gamma \rightarrow \ell \nu b \gamma$ (solid line), $\bar{t} t, t \rightarrow W b \gamma \rightarrow j j b \gamma$ (dashed line) and $\bar{t} t \gamma$ (dotted line) contributions. The additional cuts imposed are described in Sec. II. 


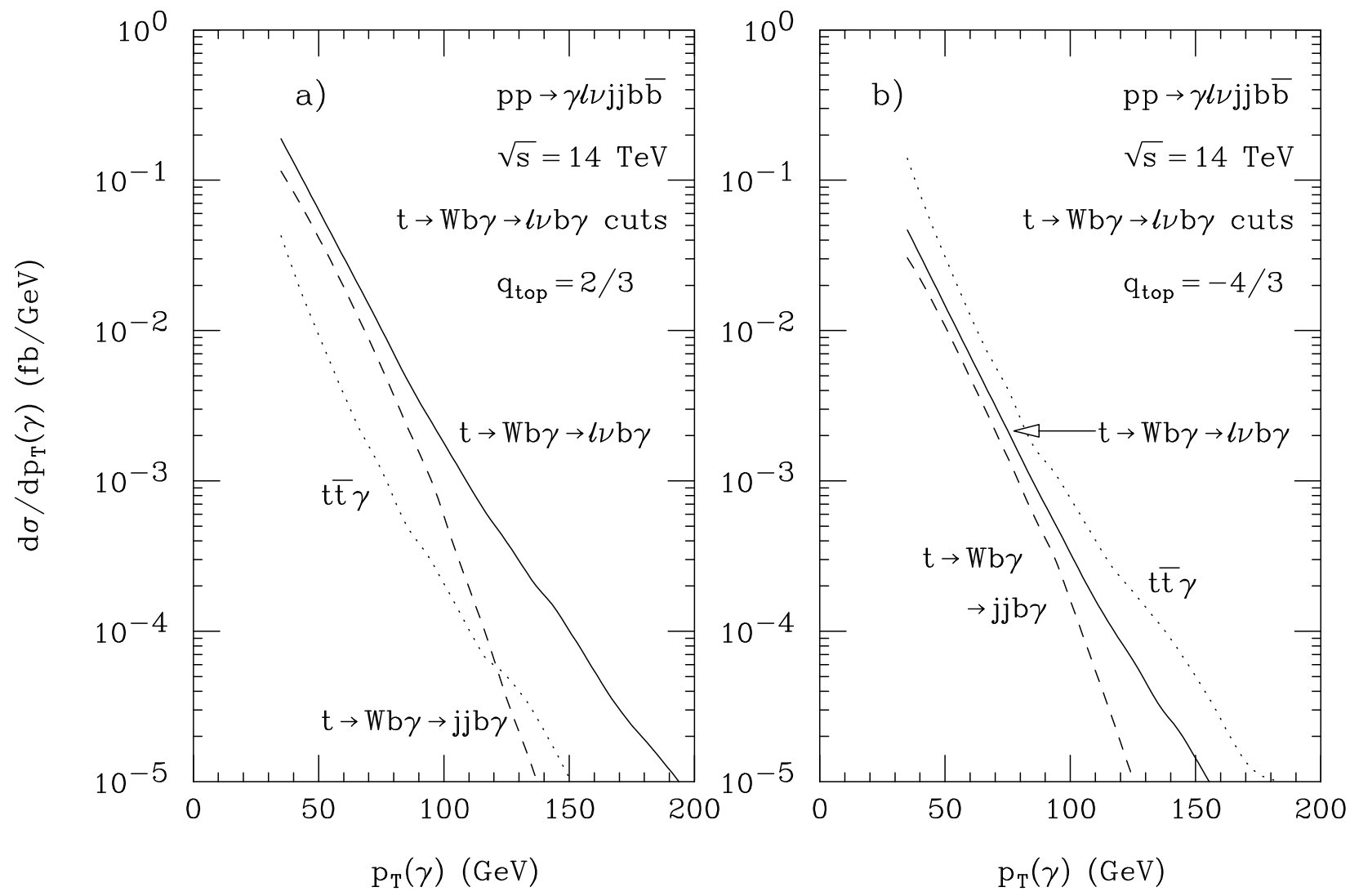

FIG. 4. The photon transverse momentum distribution for $p p \rightarrow \gamma \ell^{ \pm} \nu b \bar{b} j j$ at the LHC, imposing $t \rightarrow W b \gamma \rightarrow \ell \nu b \gamma$ selection cuts (see text), for a) $q_{t o p}=2 / 3$ and b) $q_{t o p}=-4 / 3$. Shown are the $\bar{t} t, t \rightarrow W b \gamma \rightarrow \ell \nu b \gamma$ (solid line), $\bar{t} t, t \rightarrow W b \gamma \rightarrow j j b \gamma$ (dashed line) and $\bar{t} t \gamma$ (dotted line) contributions. The additional cuts imposed are described in Sec. II. 

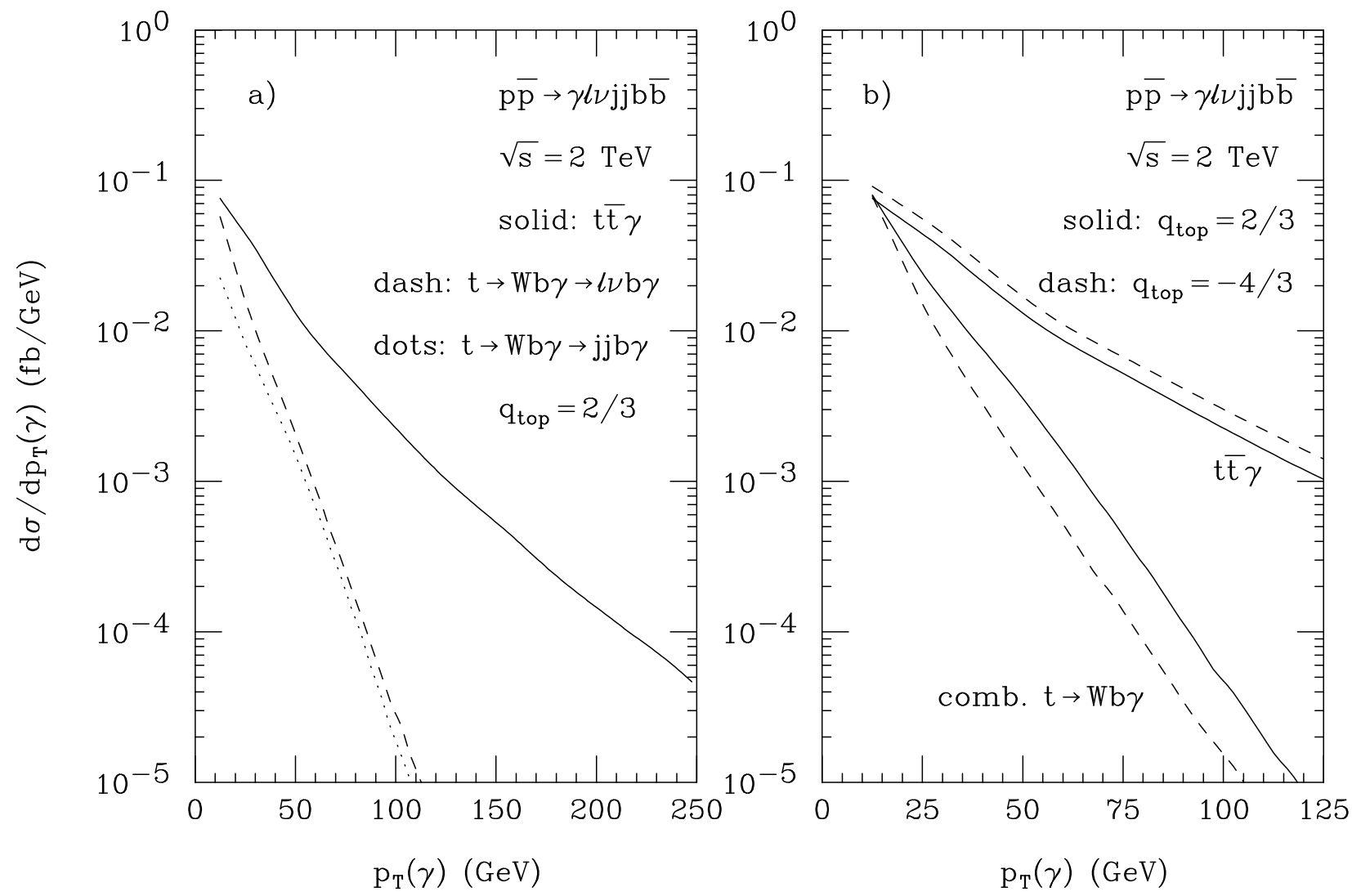

FIG. 5. The photon transverse momentum distribution for $p \bar{p} \rightarrow \gamma \ell^{ \pm} \nu b \bar{b} j j$ at the Tevatron. Part a) displays the individual contributions for $q_{t o p}=2 / 3$, imposing $\bar{t} \gamma$ (solid line), $t \rightarrow W b \gamma \rightarrow \ell \nu b \gamma$ (dashed line) and $t \rightarrow W b \gamma \rightarrow j j b \gamma$ selection cuts (dotted line). In part b), the photon $p_{T}$ distributions for the phase space region defined by the $\bar{t} t \gamma$ selection cuts and the two radiative top decay regions combined are shown for $q_{t o p}=2 / 3$ and $q_{t o p}=-4 / 3$. The cuts imposed are described in the text. 

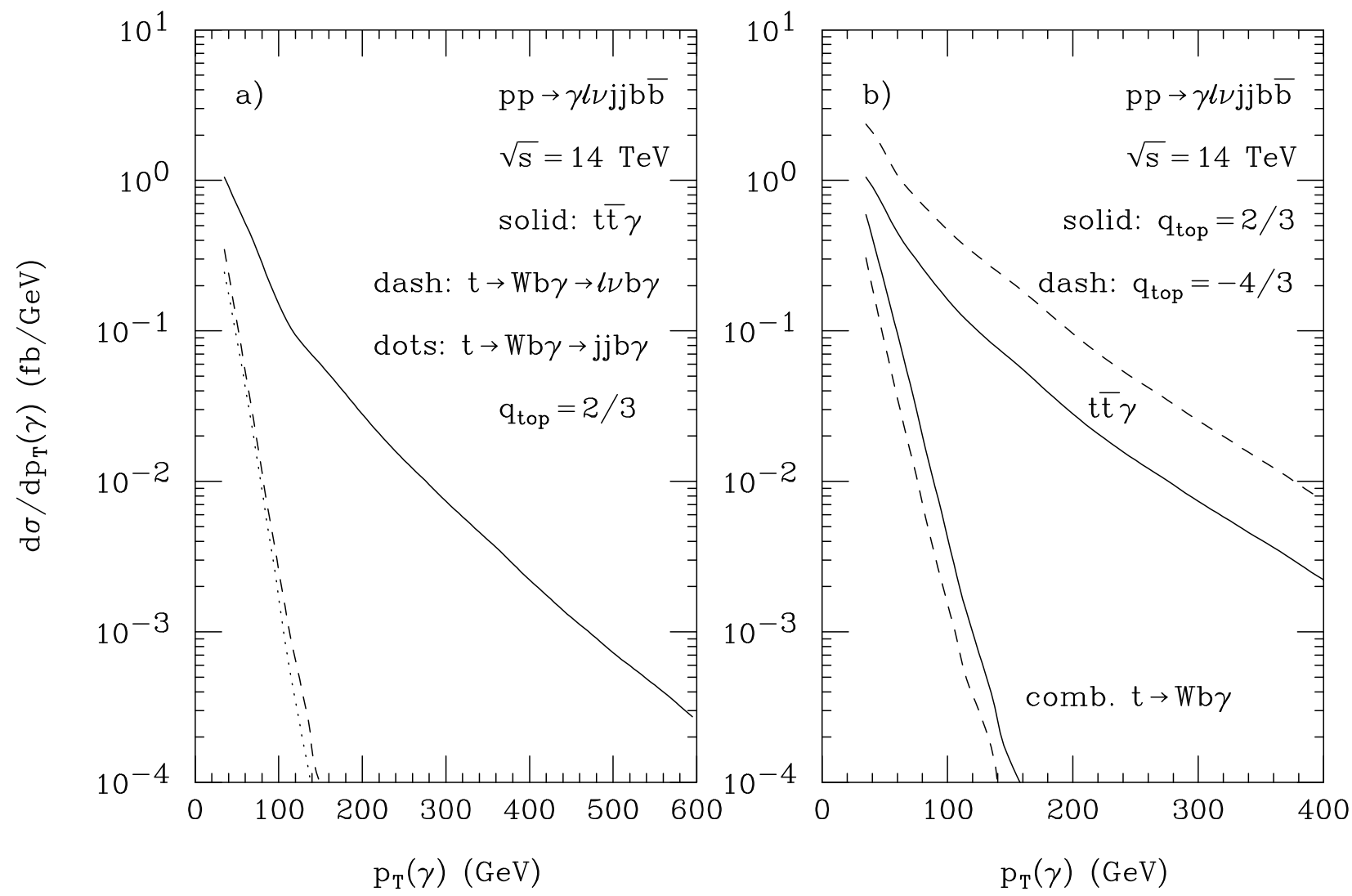

FIG. 6. The photon transverse momentum distribution for $p p \rightarrow \gamma \ell^{ \pm} \nu b \bar{b} j j$ at the LHC. Part a) displays the individual contributions for $q_{t o p}=2 / 3$, imposing $\bar{t} t \gamma$ (solid line), $t \rightarrow W b \gamma \rightarrow \ell \nu b \gamma$ (dashed line) and $t \rightarrow W b \gamma \rightarrow j j b \gamma$ selection cuts (dotted line). In part $\mathrm{b}$ ), the photon $p_{T}$ distributions for the phase space region defined by the $\bar{t} t \gamma$ selection cuts and the two radiative top decay regions combined are shown for $q_{t o p}=2 / 3$ and $q_{t o p}=-4 / 3$. The cuts imposed are described in the text. 

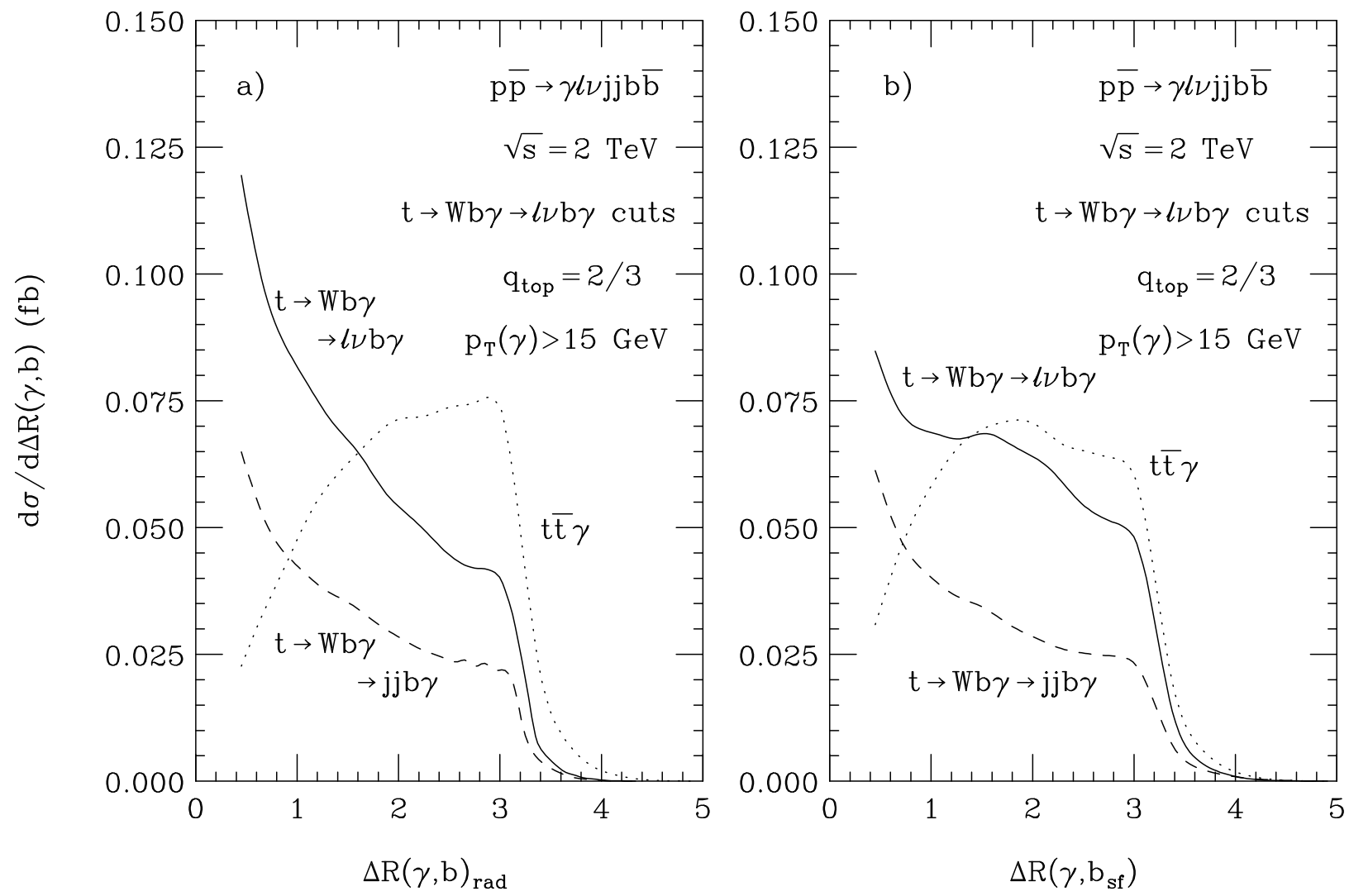

FIG. 7. Comparison of the SM $\Delta R(\gamma, b)_{\text {rad }}$ and $\Delta R\left(\gamma, b_{s f}\right)$ distributions for $p \bar{p} \rightarrow \gamma \ell \nu j j b \bar{b}$ at the Tevatron, imposing $t \rightarrow W b \gamma \rightarrow \ell \nu b \gamma$ selection cuts (see text). Shown are the contributions from $\bar{t} t$ production with $t \rightarrow W b \gamma \rightarrow \ell \nu b \gamma$ (solid lines) and $t \rightarrow W b \gamma \rightarrow j j b \gamma$ (dashed lines), and $\bar{t} t \gamma$ (dotted lines) production. The cuts described in Sec. II are imposed, except for the minimum photon transverse momentum which has been increased to $15 \mathrm{GeV}$. 

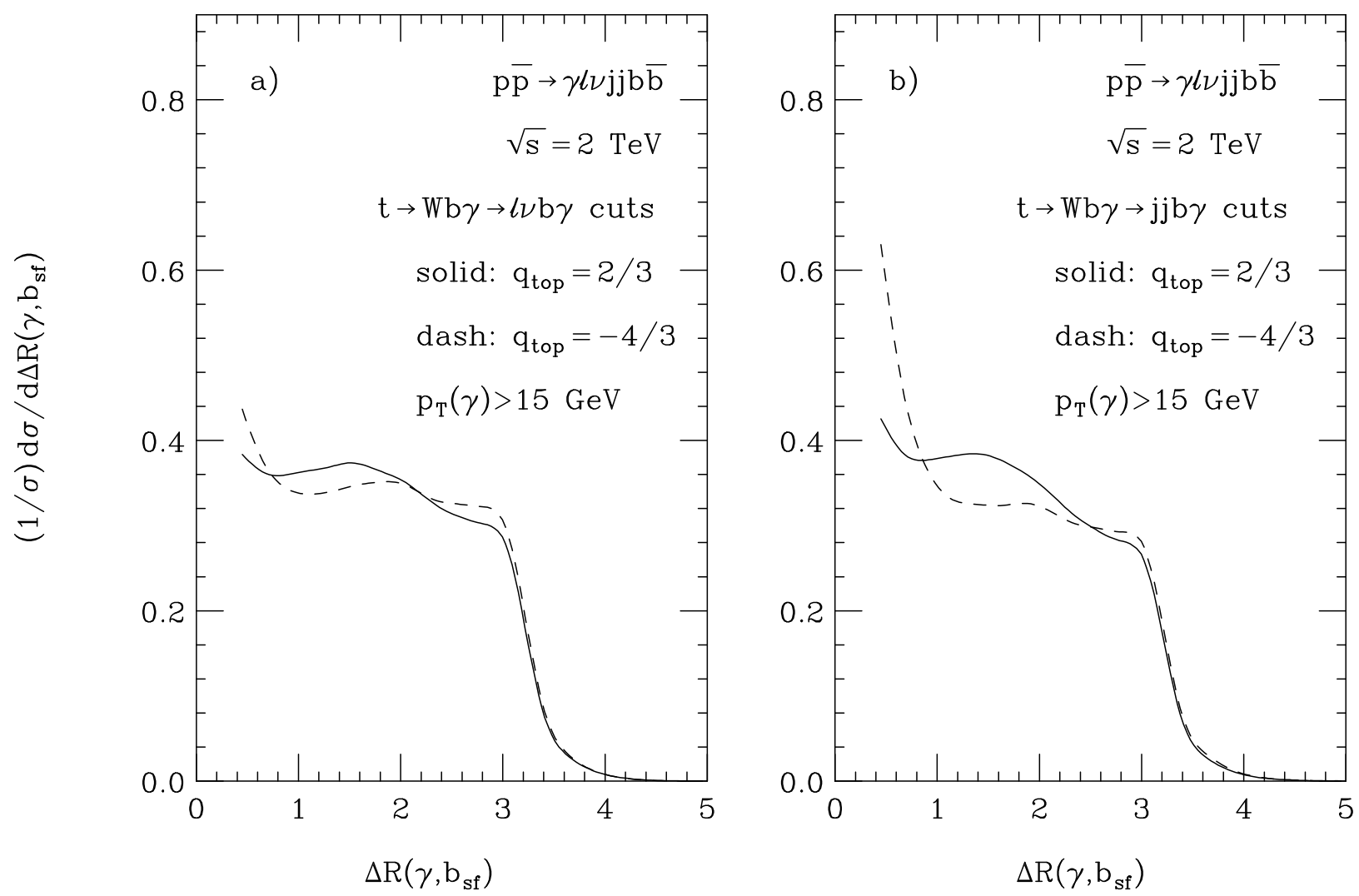

FIG. 8. The normalized $(1 / \sigma)\left(\Delta R\left(\gamma, b_{s f}\right)\right)$ distribution for $p \bar{p} \rightarrow \gamma \ell \nu j j b \bar{b}$ at the Tevatron, imposing a) $t \rightarrow W b \gamma \rightarrow \ell \nu b \gamma$ selection cuts and b) $t \rightarrow W b \gamma \rightarrow j j b \gamma$ selection cuts (see text). Shown are the results for a SM top quark charge assignment (solid lines) and for $q_{\text {top }}=-4 / 3$ (dashed lines). The cuts described in Sec. II are imposed, except for the minimum photon transverse momentum which has been increased to $15 \mathrm{GeV}$. 

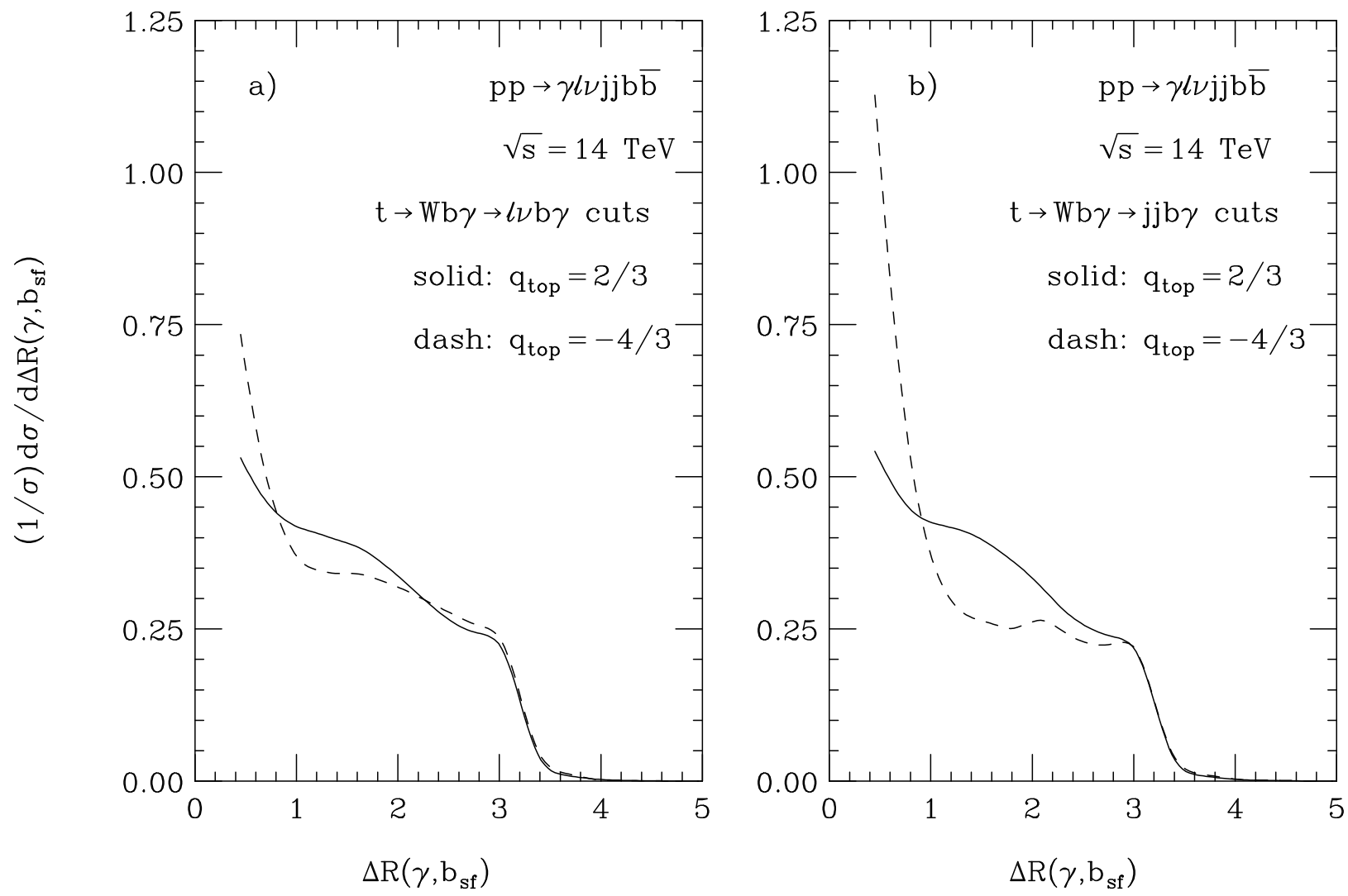

FIG. 9. The normalized $(1 / \sigma)\left(\Delta R\left(\gamma, b_{s f}\right)\right)$ distribution for $p p \rightarrow \gamma \ell \nu j j b \bar{b}$ at the LHC, imposing a) $t \rightarrow W b \gamma \rightarrow \ell \nu b \gamma$ selection cuts and b) $t \rightarrow W b \gamma \rightarrow j j b \gamma$ selection cuts (see text). Shown are the results for a SM top quark charge assignment (solid lines) and for $q_{t o p}=-4 / 3$ (dashed lines). The additional cuts imposed are described in Sec. II. 